\title{
Passaggi della Macchia Rossa di Giove
}

osservatial refrattore di om.25 nel R. Osservatorio di Palermo da Riccò.

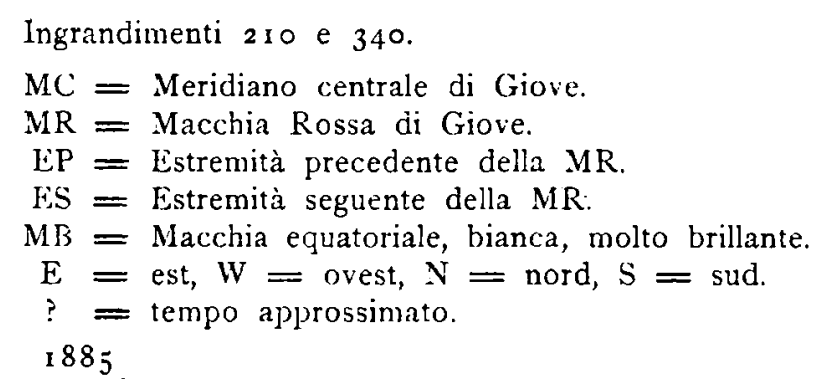

Nov. to $17^{\mathrm{h}} 37^{\mathrm{m}}$ ? Centro della MR sul MC. La MR è assai debole, specialmente nel lato $\mathrm{N}$. Nubi.

1740 Centro della MR un poco ad $W$ del MC. EP più debole dell' ES.

I 758 ES sul $\mathrm{MC}$, od un poco ad $\mathrm{E}$

18 ES un poco ad W del MC. 1886

Marzo $297^{\mathrm{h}} 3^{8^{\mathrm{m}}}$ ? ES sul MC. MR poco distinta. Immagine mediocre.

» $3_{1}^{1} 8 \quad 5^{2.5}$ Centro della MR sul MC. La MR è più debole nello interno: le punte sono un poco prolungate.

9 I 3.5 ES un poco ad E del $\mathrm{MC}$.

930.5 ES un poco ad $W$ del $M C$.

Aprile ro $739 ?$ ES prossimamente sul MC.

$74^{6} \div$ ES ad W del MC di circa $1 / 5$ del raggio del suo parallelo. La MR è distintamente colorata in rosso, specialmente al contorno $\mathrm{S}$.

Magg. 259 10.5? Centro della MR prossimamente sul MC. Immagine cattiva.

943 ? ES prossimamente sul MC.

$934 \mathrm{MB}$ sul $\mathrm{MC}$.

Giug. I $8 \quad 8 \quad 47 \quad$ EP sul MC.

9 I3 Centro della MR sul MC.

$93^{8}$ ES sul MC.

La MR è quasi bianca nel mezzo: è più lunga di $1 / 4 \mathrm{e}$ meno lunga di $1 / 3$ del diametro del suo parallelo.
Giug. $259^{\mathrm{h}} 33^{\mathrm{m}} \div$ EP sul $\mathrm{MC}$, od un poco ad $\mathrm{W}$.

$955 \div$ Centro della MR sul MC.

1030 ? ES sul MC.

$938 \div \mathrm{MB}$ sul MC.

Immagine poco distinta.

Luglio 99 ig EP sul MC.

947 : Centro della MR un poco ad $\mathrm{E}$ del $\mathrm{MC}$. 1887

Magg. $15 \quad 9^{\mathrm{h}} 6^{\mathrm{m}} ?$ ES ad W del MC di circa $1 / 2$ raggio del suo parallelo.

$\triangleright$ I 766 ? $\mathrm{EP}$ sul $\mathrm{MC}$, od un poco ad W: inmagine della MR debolissima, ineguale.

930 Centro della MR sul MC.

957 ES sul $\mathrm{MC}$.

1888

Giug. $6 \quad 8^{\mathrm{h}} 35^{\mathrm{mh}} \quad \mathrm{MB}$ sul $\mathrm{MC}$.

$851 ? E P$ un poco ad $W$ del MC. Inmagine poco distinta.

96 ? Centro della MR un poco ad E del MC.

$99 \div$ Centro della MR sul MC.

9 I5? Centro della MR appena di un poco ad $W$ del MC. I a MR è visibile con difficoltà.

935 ES sul MC: immagine un poco più distinta di prima.

Luglio 2 10 $41.5^{\circ}$ Centro della MR prossimamente sul MC. La MR si vede con difficoltà.

1 I 8.5? ES prossimamente sul MC.

I I 17.5 ? ES un poco ad $W$ del MC.

- 17836 . ES prossimamente sul MC, od un poco. ad W. I a MR è debolissima, visibile con molta difficoltà.

NB. Le osservazioni della MR in generale furono difficili, perchè la MR era poco distinta.

In seguito si pubblicheranno le osservazioni complete ed $i$ disegni relativi del 1885 al 1888 .

Palermo 1889 Aprile.

A. Riccò.

\section{Schreiben von Herrn Prot. R. Wolf in Zuirich an den Herausgeber \\ betr. den Vorübergang des Cometen $1826 \mathrm{~V}$ vor der Sonnenscheibe.}

Die Notiz von Professor Galle in A. N. 2912 erinnert mich daran, dass ich im Jahrgange 30 der Vierteljahrsschrift der naturforschenden Gesellschaft in Zürich zwei sich auf jenen Cometen-Durchgang bezügliche Briefe veröffentlicht habe, welche Gambart 1826 Nov. 6 und 1826 Nov. 22 an Gautier in Genf schrieb. Sie wiurden wohl einzelnen unserer Fachgenossen einen Dienst erweisen, wenn Sie in den A. N. auf diese Publication hinweisen wollten. 